\title{
Auditory Brainstem Implants
}

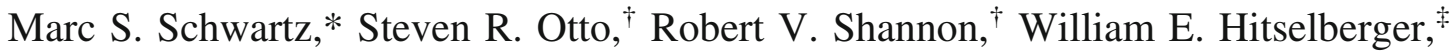 \\ and Derald E. Brackmann* \\ *House Clinic and ${ }^{\dagger}$ House Ear Institute, Los Angeles, California 90057, and ${ }^{\ddagger}$ St. Vincent’s Hospital, Los Angeles, \\ California 90057
}

\begin{abstract}
Summary: The development of cochlear implantation has allowed the majority of patients deafened after the development of language to regain significant auditory benefit. In a subset of patients, however, loss of hearing results from destruction of the cochlear nerves, rendering cochlear implantation ineffective. The most common cause of bilateral destruction of the cochlear nerves is neurofibromatosis type 2 (NF2). The hallmark of this genetic disorder is the development of bilateral acoustic neuromas, the growth or removal of which causes deafness in most patients. Patients with NF2 may benefit from direct stimulation of the cochlear nucleus. We describe the devel-
\end{abstract}

opment, use, and results of the auditory brainstem implant (ABI), which is typically implanted via craniotomy at the time of tumor removal. Most patients with the implant have good appreciation of environmental sounds, but obtain more modest benefit with regard to speech perception. The majority of patients make use of the implant to facilitate lip reading; some can, to varying degrees, comprehend speech directly. We discuss future directions in central implants for hearing, including the penetrating $\mathrm{ABI}$, the use of $\mathrm{ABI}$ in nontumor patients, and the auditory midbrain implant. Key Words: Auditory brainstem implant, deafness, neurofibromatosis type 2 , acoustic neuroma, vestibular schwannoma.

\section{INTRODUCTION}

Patients with neurofibromatosis type 2 (NF2) develop bilateral acoustic neuromas. Growth or removal of these tumors typically results in deafness due to disruption of the cochlear nerves, and because of this disruption patients with NF2 are generally not candidates for cochlear implantation. The auditory brainstem implant (ABI) was developed to bypass the auditory nerve and to directly stimulate the cochlear nucleus complex. ${ }^{1}$

ABIs share a design concept with cochlear implants, in that both exploit the frequency tuning of neurons in the cochlea and the cochlear nucleus complex and the tonotopic organization of these structures. Nonetheless, even though useful auditory sensation is provided by the ABI, the results with this device do not usually match the exceptional results seen with cochlear stimulation., ${ }^{2,3}$ Also, because of variations in brainstem anatomy, electrode placement, and stimulation patterns seen with ABIs, their programming is significantly more complex than for cochlear implants. ${ }^{4}$

Address correspondence and reprint requests to: Marc S. Schwartz, M.D., House Clinic, 2100 West Third St., Los Angeles, CA 90057.

E-mail: mschwartz@hei.org.
More than 230 patients have undergone implantation of the ABI at the House Ear Institute (Los Angeles, CA) since 1979. The first 25 patients received a single-channel device. Since 1992, a multichannel ABI has been used, with 71 patients receiving an 8-electrode array and the latest group receiving a 21-electrode array. In 2000, a multichannel ABI received U.S. Food and Drug Administration (FDA) approval for commercial release (the Nucleus system; Cochlear Corp., Englewood, CO). Currently, a multichannel penetrating array is in development and nearing the stage for limited clinical trials in humans. This device is designed to increase the precision of electrical stimulation within the cochlear nucleus complex.

\section{DEVELOPMENT OF THE ABI}

The first ABI was a simple ball-type electrode implanted in an NF2 patient following removal of an acoustic neuroma by William E. Hitselberger and William F. House in $1979 .{ }^{5}$ Used with a modified, body-worn hearing aid, this device provided useful auditory sensation. Based on experiences with this initial patient, a twoelectrode (and later three-electrode) mesh-type array was developed by the Huntington Medical Research Institute (Pasadena, CA). These devices were used in combination 


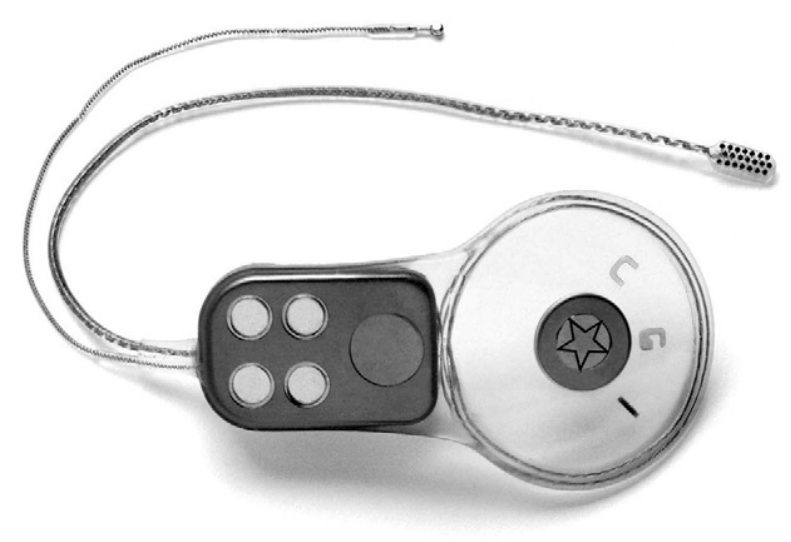

\section{PATIENT SELECTION AND COUNSELING}

The hallmark of NF2 is the development of bilateral acoustic neuromas. Patients may also develop other tumors, including other schwannomas, meningiomas, and gliomas, throughout the neuroaxis. Modern treatment modalities have significantly decreased the mortality associated with these tumors. A major goal in the treatment of NF2 is the preservation of hearing via early diagnosis and treatment of acoustic neuromas. Nevertheless, the majority of patients with NF2 will progress to bilateral deafness despite all attempts to prevent this. ${ }^{8}$ In this group of patients, the auditory information provided by

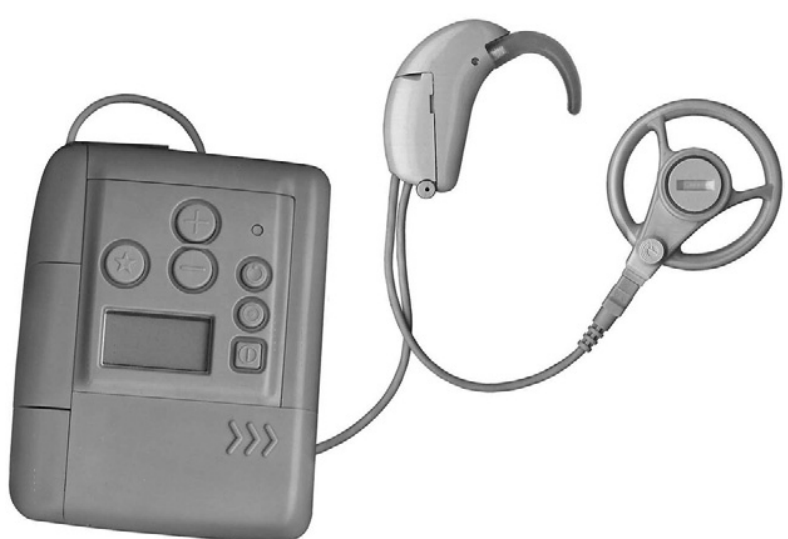

FIG 1. Top: Implanted portion of the auditory brainstem implant $(\mathrm{ABI})$, including receiver-stimulator, 21-electrode array, and balltype remote ground electrode. Bottom: Externally worn apparatus, including postauricular microphone, speech processor, and transmitter coil.

with modified 3M-House type cochlear implant processors. ${ }^{6}$

In conjunction with Huntington Medical Research Institute and Cochlear Corporation, the 8-electrode and 21-electrode arrays were developed after experiments suggested the feasibility of multichannel stimulation. The current 21-electrode system (Nucleus 24 ABI system, or ABI24) consists of $0.7-\mathrm{mm}$ platinum disk electrodes aligned on a flexible silicone and mesh backing (FIG. 1). The ABI24 system includes a microphone headset, the Nucleus SPrint sound processor, and a transmitter coil. The processor, also used with cochlear implantation, uses the Nucleus SPEAK spectral peak speech coding strategy.

In patients with NF2, continuous follow-up with magnetic resonance imaging (MRI) is critical; however, because the ABI is MRI compatible (at $1.5 \mathrm{~T}$ ) only with removal of the magnet from the receiver-stimulator, use of the supplied magnet is impractical. ${ }^{7}$ During the implantation process, therefore, the magnet used to couple the transmitter to the implanted receiver for the typical cochlear implant patient is removed for NF2 patients, and the external transmitter coil is always attached to the scalp using an adhesive disk. the ABI can provide significant benefit in terms of sound perception.

The multichannel $\mathrm{ABI}$ is approved for use in patients with NF2 who are at least 12 years old and have language competency. Implantation can be done at the time of first- or second-side tumor removal. Because a small but significant percentage of patients $(8 \%$, at the House Ear Institute) fail to respond to stimulation by the ABI, firstside implantation allows a second opportunity for implantation if necessary. We have adopted the strategy of first-side implantation even if serviceable hearing remains on the contralateral side.

Preoperative counseling and informed consent is critical for patients undergoing ABI placement. Because acute hearing loss often occurs at the time of surgery, and because results with the device are highly variable, patients must have realistic expectations. Even more important is an appreciation of the prolonged nature of the follow-up evaluation and device tuning necessary to optimize ABI performance.

Although difficult to quantify, patient motivation and willingness to actively participate are important factors in reaching maximal efficacy. During the programming process, numerous factors may conspire to prevent device acceptance and use. These include initially suboptimal auditory results and the generation of nonauditory responses (e.g., tingling, dizziness) with ABI activation. Patients must comprehend these possibilities ahead of time and be willing to work through them. Patients may be reassured that, with time and with their cooperation, these problems typically improve.

\section{SURGICAL CONSIDERATIONS}

The ventral and dorsal cochlear nuclei are the targets for placement of the ABI electrode array. These structures lie along the dorsolateral surface of the brainstem at the lateral recess and may be accessed via the foramen of Lushka, which denotes the terminus of the recess. Normally, these structures are not immediately visible via the standard surgical approaches, although several im- 


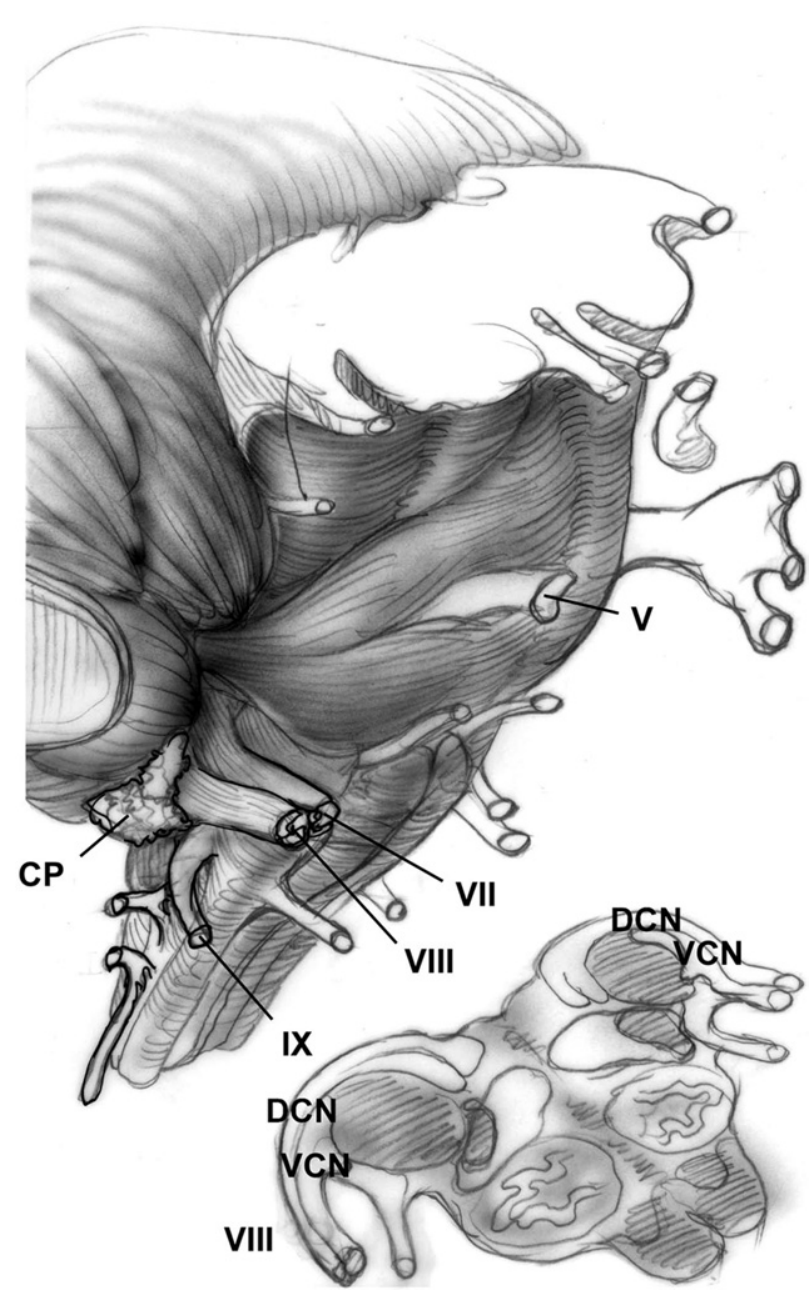

FIG 2. Schematic view of the brainstem at the pontomedullary junction. External view and cross-section at the cochlear nucleus complex are shown. Relevant cranial nerves are labeled with roman numerals. CP, choroid plexus; DCN, dorsal cochlear nucleus; VCN, ventral cochlear nucleus.

portant landmarks indicate their location. The relevant anatomy of the brain at the pontomedullary junction is shown in Figure 2.

As the lateral foramen is approached, it may be hidden by the flocculus of the cerebellum. The entrance of the recess is marked by the choroid plexus, which usually extends though the foramen from the fourth ventricle. The lateral recess is also defined by the eighth cranial nerve, the root of which forms its superior wall, and by the ninth cranial nerve, which exits the brain along its inferior edge. Notably, however, the normal anatomy in this region is often distorted by the presence of an overlying acoustic tumor, making identification of these structures significantly more difficult.

The ventral cochlear nucleus serves as the main relay between the afferent cochlear input and the ascending auditory pathways. As such, it is the main target for stimulation by the electrode array. This nucleus lies at the most lateral portion of the lateral recess (at the fo- ramen of Lushka) superiorly and slightly anteriorly. It may be useful to think of the foramen as a clock-face with 12 o'clock superiorly. For right-sided implantation, the nucleus is found at about 1 o'clock. Once again, however, distortion by tumor may alter this relationship.

In our series, the surgical approach for implantation of $\mathrm{ABI}$ has been exclusively via the translabyrinthine route. We feel that this approach provides the most direct access to the site of the cochlear nuclei and the best visualization of the relevant anatomy. ${ }^{9}$ The retrosigmoid approach has also been successfully used for ABI implantation. ${ }^{10}$ Surgery for resection of the overlying acoustic neuroma is performed in the standard fashion. An array of neurophysiologic monitoring is used during ABI implantation. In addition to facial nerve monitoring, electromyographic monitoring of the glossopharyngeal nerve is accomplished with placement of a recording electrode in the soft palate. In addition scalp electrodes are placed to monitor evoked auditory brainstem responses (EABRs).

At the time of electrode array placement, proper positioning is confirmed intraoperatively. ${ }^{11}$ Various electrodes on the array are stimulated via the receiver coil and responses are recorded. Interpretation of the EABRs requires an experienced electrophysiologist, who provides feedback to the surgeon. Monitoring of the facial and glossopharyngeal nerves is performed simultaneously, to detect undesirable stimulation of these nerves.

\section{OPERATIVE AND PERIOPERATIVE CARE}

The operative technique for translabyrinthine resection of acoustic neuroma has been described previously. Thorough understanding of this approach is critical. For ABI implantation, the initial scalp incision is altered slightly to accommodate subgaleal placement of the receiver-stimulator (FIG. 3). Thereafter, the usual steps in tumor resection are performed, including tumor resection with efforts made to preserve the facial nerve. Maintenance of a recognizable stump of the vestibulocochlear nerve is useful for localizing the lateral recess.

After preparing the exterior surface of the skull to properly seat the receiver, the electrode array is carefully inserted into the lateral recess overlying the presumed site of the cochlear nucleus complex (FIG. 4). It is our practice to insert nearly the entire length of the array into the recess. The device is designed to maintain a stable position at the lateral foramen itself. After good positioning is attained, teased Teflon felt (polytetrafluoroethylene; DuPont) is packed behind the array, to further reinforce its stability.

Next, testing is performed to confirm the presence of EABRs, and to detect stimulation of adjacent cranial 


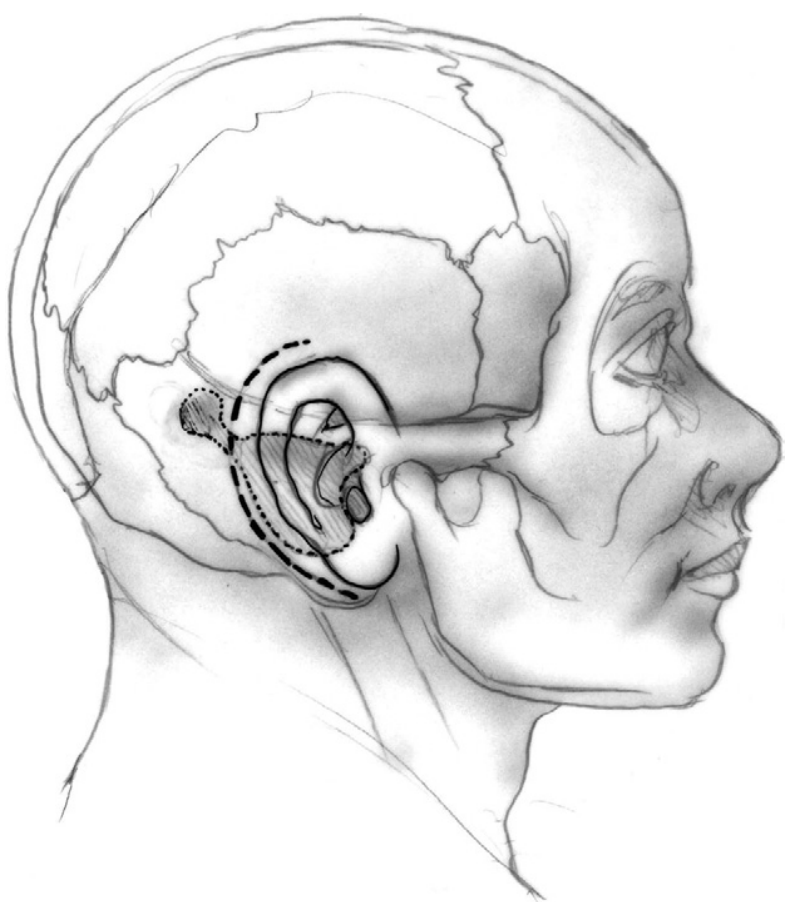

FIG 3. Location of the incision for translabyrinthine craniotomy for resection of acoustic neuroma with insertion of ABI. The superior portion of the incision is carried wider and higher than would be typical for tumor resection alone.

nerves or changes in vital signs. Stimulation is administered via electrodes at the four corners of the array, and the presence or absence of EABRs at each location is used to guide any repositioning that may be necessary (FIG. 5). After adequate positioning of the electrode array has been achieved, the magnet is removed from the receiver-stimulator and replaced with a nonmagnetic spacer. The receiver-stimulator is then carefully positioned in its bony trough and secured, the ground electrode is placed in the temporalis muscle, and the wound is closed.

The initial postoperative care of patients undergoing ABI placement differs little from that of the typical patient undergoing craniotomy for resection of acoustic neuroma alone; however, because these patients have NF2, other issues may be of concern. Most notably, in the presence of pathology affecting the contralateral lower cranial nerves, careful assessment of airway protection and swallowing is mandatory. We have noted a far higher incidence of aspiration and dysphagia in this group of patients than in the acoustic neuroma population as a whole. The risk of cerebrospinal fluid leak and meningitis may also be increased in this group of patients, likely related to wicking of fluid along the electrode cable extending from the subarachnoid space to the scalp. Most patients are successfully treated for leakage with pressure dressing or lumbar spinal drainage. Two patients early in the series, however, required device explantation due to abscess formation.
Unlike cochlear implants, the external ABI transmitter coil is not held in place using an implanted magnet. To achieve satisfactory positioning, the area overlying the receiver antenna is shaved, and an adhesive retainer disk, containing a small metal tab, is placed. The transmitter coil magnet adheres to this tab. Application on the scalp of a small tattoo has been useful for some patients in helping to guide consistent transmitter placement.

\section{DEVICE ACTIVATION AND PROGRAMMING}

Initial stimulation using the ABI is performed 6-8 weeks after implantation. Programming of the device follows some of the same steps as are used with cochlear implants. Unlike cochlear implants, however, with the ABI the pitch percepts elicited by the various electrodes are highly variable. This significantly increases the complexity of the programming process. Furthermore, there is the possibility of generating nonauditory responses to stimulation, another factor that requires time for management. Nonauditory sensation is thought to be due to the spread of electrical stimulation outside the cochlear nucleus complex. ${ }^{12,13}$ Given the possibility of eliciting changes in vital signs, initial stimulation is always administered with emergency medical assistance readily available.

Initial stimulation tasks include setting of threshold and comfort levels, evaluation and management of nonauditory stimulation, and pitch scaling. Monopolar stimulation is administered via each electrode, and data are collected regarding the sound quality of auditory response (including level and pitch) and of nonauditory sensations. To minimize nonauditory responses while maintaining sound quality, stimulation parameters can be adjusted. Stimulating in bipolar mode may also serve to eliminate or minimize nonauditory sensation. Occasionally, it is necessary to deactivate individual electrodes because of annoying nonauditory responses.

Next, electrodes that elicit auditory responses are pitch scaled. Because of variations in individual anatomy and electrode array positioning, this process is generally not straightforward and requires significant time. This process is also highly subjective, and patients may have difficulty assigning pitch values to the electrodes. Often, patients are better able to qualitatively describe auditory response (e.g., bell-like, drum-like) than to describe a specific pitch. To help with pitch assignments, pairwise electrode comparisons are performed. After comparing various pairs of electrodes, the electrodes can be ordered.

The results of pitch ranking are next further refined by eliminating electrodes that do not provide distinct auditory sensations and by filling pitch gaps by combining stimulation between low- and high-sounding electrodes. A processor map is configured and the device is programmed. Among patients who do achieve successful 


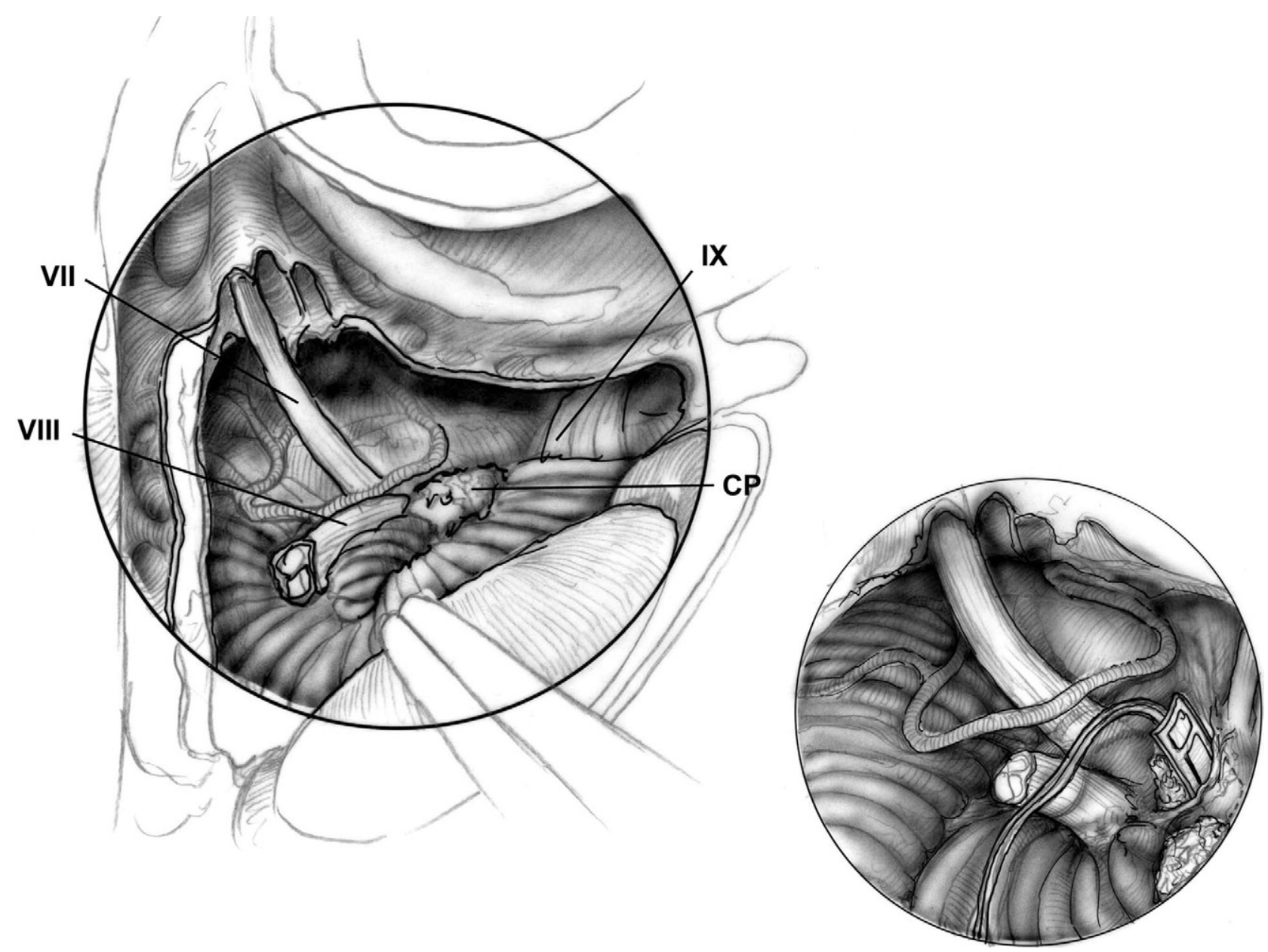

FIG 4. Left: Surgical view of the cerebellopontine angle via translabyrinthine craniotomy after removal of acoustic neuroma. Right: Site of insertion of $\mathrm{ABI}$ electrode array via lateral foramen. The electrode array must be rotated to the superior portion of the lateral recess (adjacent to the stump of the cochlear nerve) from the position shown. Relevant cranial nerves are labeled with roman numerals. CP, choroid plexus.

auditory response over multiple electrodes, pitch responses generally follow three possible patterns. One group of patients generally perceives high pitch medially and low pitch laterally. A second group perceives pitch in a flattened pattern across the array. A third group shows a random pattern of pitch change. Although a distinct pitch gradient may predict improved performance, this factor does not reach statistical significance. ${ }^{3}$ As is seen in cochlear implantation, other factors can significantly influence performance and benefit.

At the time of initial stimulation, patients may be dissatisfied with the quality of their auditory sensation; however, they can be assured that with time and experience using the ABI, sound quality can be expected to improve and any remaining nonauditory stimulation can be expected to generally ease. Also, reprogramming is done at each follow-up interval, resulting in further improvements.

\section{SOUND AND SPEECH PERCEPTION MEASURES AND RESULTS}

Medical and audiologic follow-up is conducted every 3 months for the first year, and annually thereafter. Although the schedule was initiated in preparation for FDA clinical trial, we think that this intensive schedule also provides significant benefit with regard to optimizing ABI performance. Changes in perceptual responses to stimulation over time have been frequently noted with ABIs. Certainly, changes occur at a much higher rate than with cochlear implants. ${ }^{3}$

At each follow-up visit, an extensive battery of audiologic tests is performed. Testing includes the SERT, ${ }^{14}$ the MTS test, ${ }^{15}$ the NU-CHIPS test, ${ }^{16}$ and the CID sentence test, ${ }^{17}$ all administered in sound-only mode. The Iowa consonant and vowel tests ${ }^{18}$ and the CUNY sentence test ${ }^{19}$ are administered with sound only, vision 

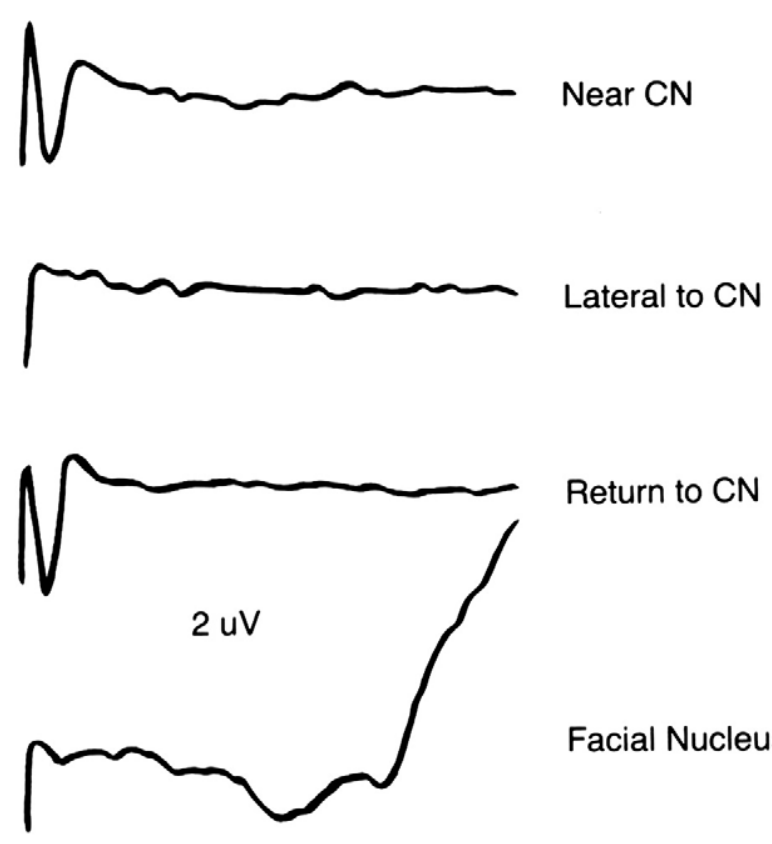

Return to $\mathrm{CN}$

Facial Nucleus

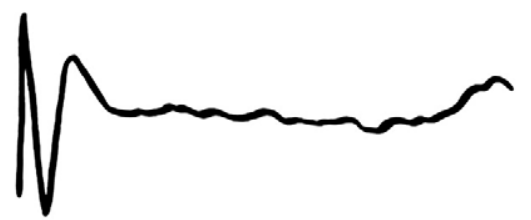

Return to $\mathrm{CN}$

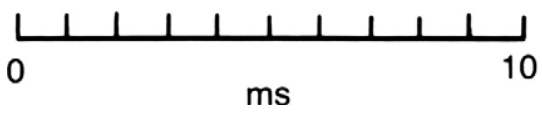

FIG 5. Tracings of intraoperative evoked auditory brainstem responses. $\mathrm{CN}$, cochlear nucleus.

only, and sound plus vision modes, to assess use of the $\mathrm{ABI}$ in combination with lip reading.

Results with the 8-channel electrode array have pre- viously been reported, most recently by Otto et al. ${ }^{3}$ This report, the data from which were also used in gaining FDA approval, showed significant benefits for ABI recipients undergoing $\mathrm{ABI}$ implantation. In this report, the majority of patients scored significantly above chance on several closed-set speech perception tests when tested with sound alone. Significant comprehension of sentence material presented in sound only was unusual. As an adjunct to lip reading, significant benefit was seen with both closed-set and open-set testing. In this series, 24\% of individual electrodes could not be used because of nonauditory stimulation. Nonauditory sensations were usually described as tingling in the head or body. A smaller number of patients described dizziness or slight jittering of vision. This report also detailed the improvement seen over time with continuous ABI use. Mean MTS test results improved from $26 \%$ at 3 months to $78 \%$ at 6 years. $^{3}$

We have analyzed a recent consecutive series of 86 patients undergoing ABI implantation. This series includes implantation of both 8-electrode and 21-electrode arrays. Upon initial analysis of data, no significant differences were seen on audiologic testing between these devices; however, the 21-electrode array may provide electrode-specific pitch sensations that contribute to sound recognition and may allow better flexibility to avoid nonauditory stimulation.

Of these 86 patients, results are reported here for 60 patients at least 6 months following implantation. Of the remaining 26 patients, 1 was explanted, 2 were not activated, 13 did not respond to stimulation (nonresponders), and 10 did not have adequate follow-up. Figure 6 shows overall results for audiologic tests administered with sound alone, and Figure 7 shows results for tests administered with sound alone, vision alone, and

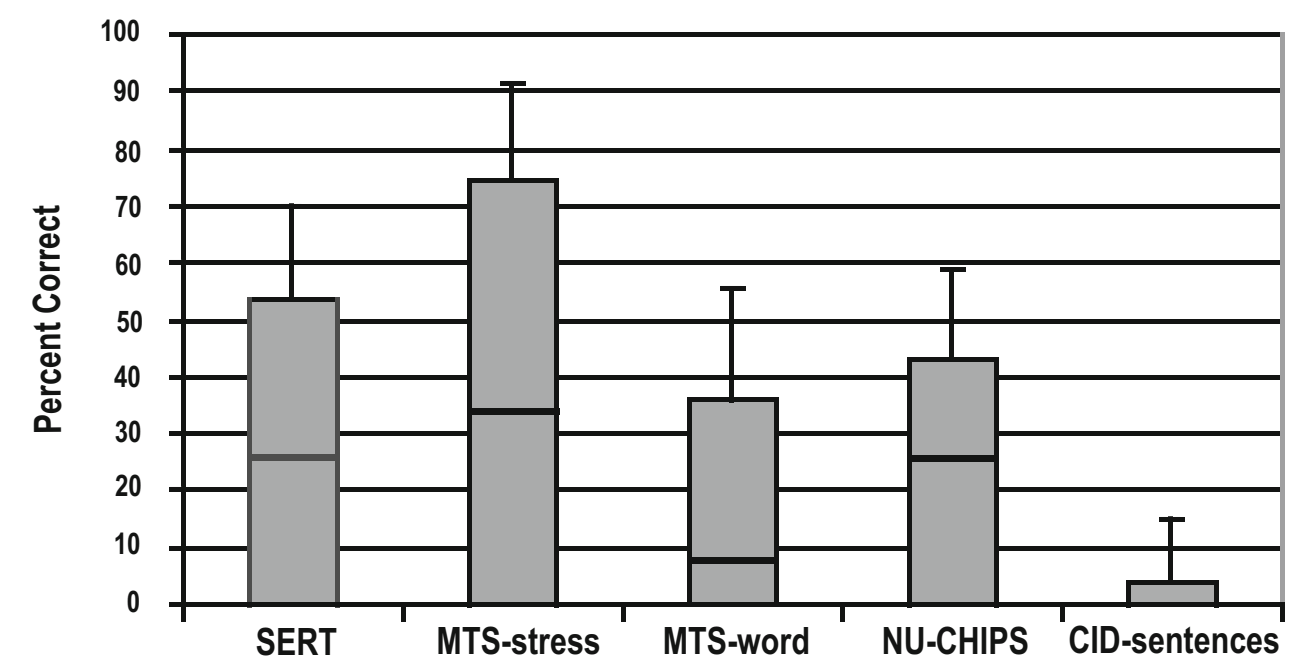

FIG 6. Results of audiologic testing of ABI for sound alone. Tests include closed-set sound (SERT), ${ }^{14}$ closed-set speech (MTS-stress, MTS-word, NU-CHIPS), ${ }^{15,16}$ and open-set speech (CID-sentences). ${ }^{17}$ Solid horizontal lines indicate chance response for closed-set tests. Error bars indicate standard deviation. 


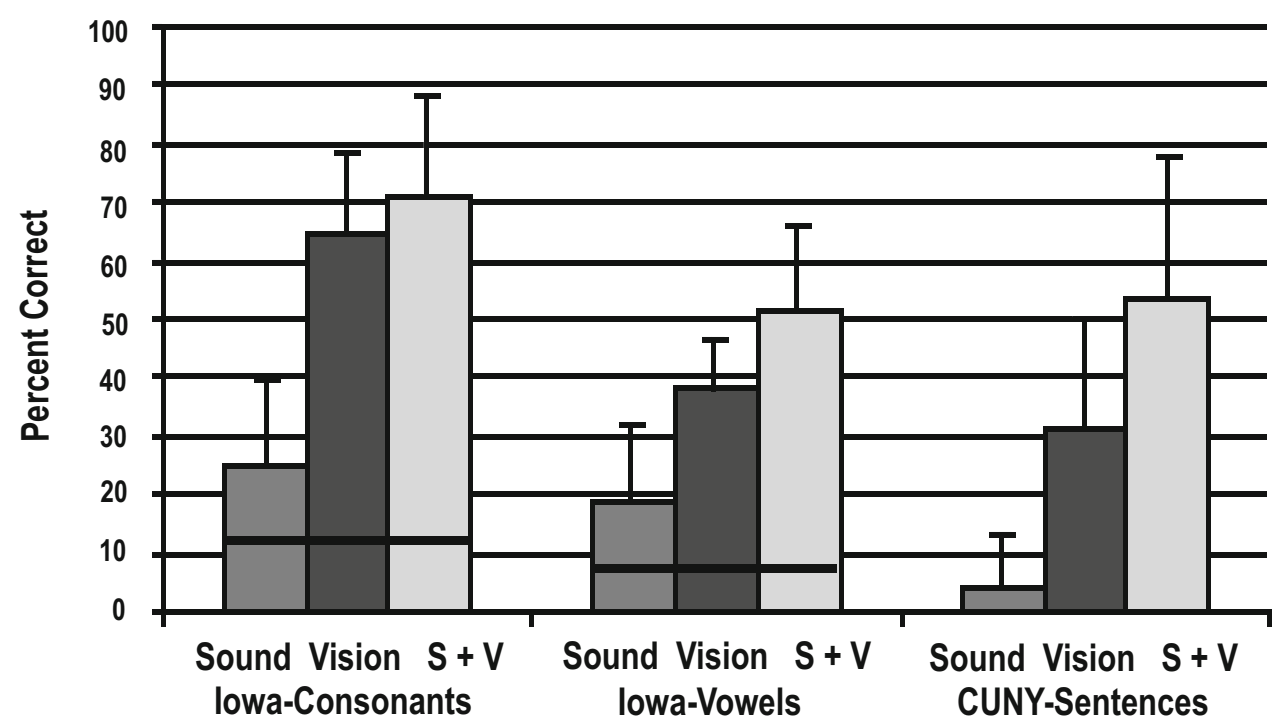

FIG 7. Results of audiologic testing of $A B I$ to augment lip reading, involving both sound (S) and vision (V). Tests include closed-set speech (lowa-consonants, lowa-vowels) ${ }^{18}$ and open-set speech (CUNY-sentences). ${ }^{17}$ Solid lines indicate chance response for closedset tests. Error bars indicate standard deviation.

sound plus vision. The magnitude of the improvement over the expected score for chance (or guessing) can be appreciated. The utility in combination with lip reading can also be appreciated; however, the mean improvements seen represent a wide range when comparing individual patients (FIG. 8).

Speech perception results with ABI do not typically match the very high levels seen in modern cochlear implantation. When used in combination with lip reading, however, the auditory sensations provided by ABI can be very useful in facilitating oral communication. With regular and continuous use, significant improvement in performance, continuing for up to a decade, is seen with most patients. Proper preoperative counseling and realistic expectations are important to prepare patients about the necessity for perseverance in this regard (FIG. 9).

\section{FUTURE DIRECTIONS}

Although ABI provides substantial auditory benefit to patients with NF2 deafened by destruction of the cochlear nerve, for most patients the results are by no means outstanding. Several new endeavors are currently being undertaken with the goals of both improving hear-

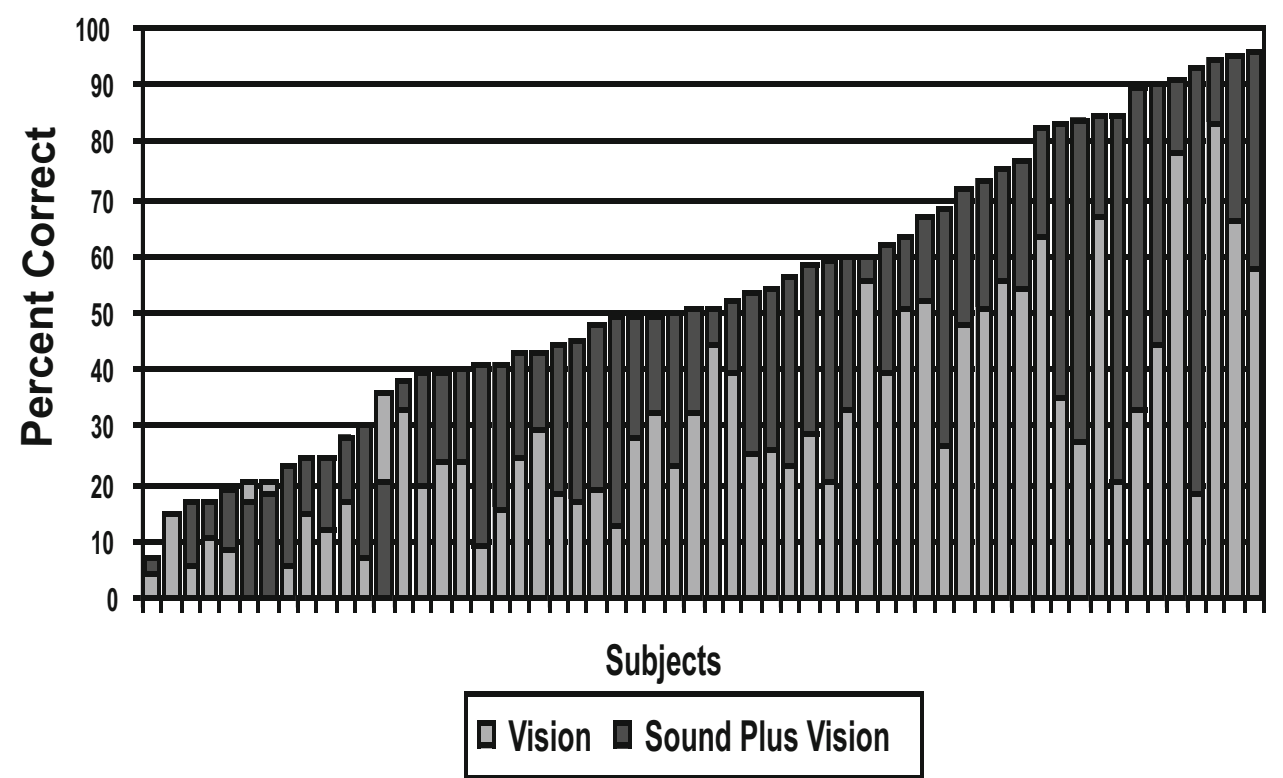

FIG 8. Results of CUNY-sentences test, ${ }^{17}$ by individual patient. Results are provided for vision alone (light portion of bars) and for vision plus sound (total height of bars, with additional benefit of $A B I$ represented by the dark portion). 


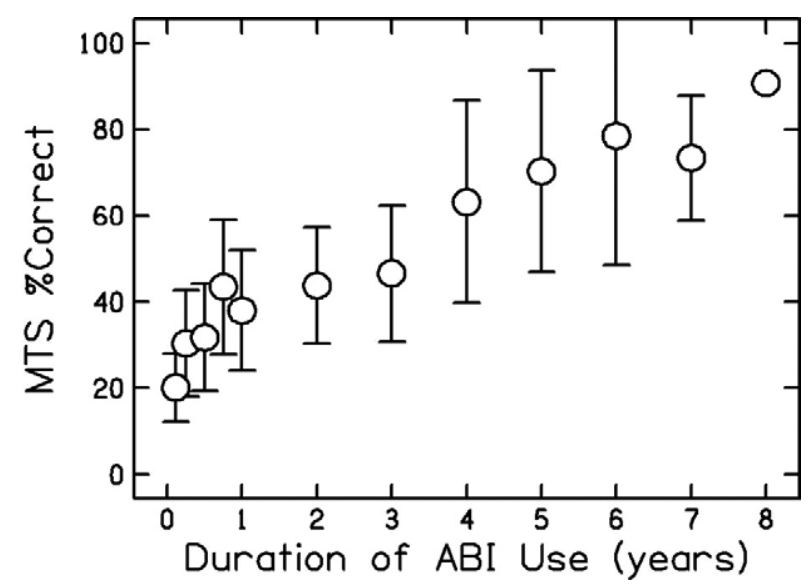

FIG 9. Improvement in MTS-word ${ }^{15}$ testing over time. Error bars indicate the $95 \%$ range of data for each point. The number of subjects decreases over time from 61 at the initial testing to a single subject at 8 years.

ing results in the core group of patients and expanding indications for the device.

\section{PENETRATING MICROELECTRODE ARRAY}

As the findings discussed here demonstrate, patients with NF2 receiving ABIs generally do receive significant and measurable benefit with regard to auditory sensation and enhancement of lip reading. For many patients, however, these benefits remain modest. Animal studies have shown the feasibility and effectiveness of implanting penetrating electrodes in the cochlear nucleus complex. ${ }^{20-22}$ With stimulation within the substance of the nuclear complex, lower thresholds and wider dynamic ranges were found. Further research has established that penetrating electrodes can be safely inserted without significant injury to tissue or blood supply in long-term preparations. $^{23}$

A penetrating microelectrode array has been developed jointly by the House Ear Institute, Huntington Medical Research Institute, and Cochlear Corporation. This array is designed to access the cochlear nucleus complex when inserted using a specifically designed spring-powered tool to penetrate the ependyma and assure on-axis insertion of the electrodes. Theoretically, the penetrating array will be better able to capitalize on the tonotopic gradient, which extends into the matrix of the nucleus. Following the successful completion of animal experiments, the penetrating array received clearance from the FDA for initial use in humans. This trial started in 2003 and recently concluded (in 2007).

\section{ABI IN NONTUMOR PATIENTS}

At the current time in the United States, ABIs are approved only for use in patients with NF2 concurrently undergoing craniotomy for resection of acoustic neuro- mas. Given the relatively modest audiometric gains seen in this group of patients, craniotomy with the express purpose of ABI placement has not been considered sufficiently beneficial to justify the procedure's inherent risks. In Europe, however, ABI placement has been performed in a select group of patients without tumors. This group of patients includes deafened people who are not candidates for cochlear implantation. Indications include congenital cochlear nerve dysgenesis, cochlear calcification, and cochlear nerve damage related to meningitis or trauma. ABI is of benefit only in patients who were postlingually deafened or in young children still able to develop spoken language (similar to cochlear implant).

Notably, the group of patients implanted in the absence of acoustic neuromas has shown significantly greater auditory benefit than those with these tumors. Although the reason for this difference between tumor and nontumor patients is unknown, hypotheses include subclinical brainstem injury related to tumor growth or resection and intrinsic maldevelopment of the cochlear nucleus related to the NF2 mutation.

Colletti and Shannon ${ }^{24}$ recently reported results in 10 nontumor patients implanted via retrosigmoid craniotomy. Approximately half of adult patients achieved outstanding audiometric results, including open-set recognition with speech alone. These results are comparable to those obtained with cochlear implantation. The results with children are preliminary and more difficult to interpret; however, a significant proportion of children do seem to obtain some auditory benefit. ${ }^{25}$

\section{AUDITORY MIDBRAIN IMPLANT}

Early studies have also begun on the feasibility of placing an electrode array at the level of the inferior colliculus. The inferior colliculus lies downstream from the cochlear nucleus along the central auditory pathway and shares with this structure a tonotopic organization. Implantation at the level of the auditory midbrain has the advantage of bypassing the cochlear nucleus at the pons, which may be damaged by tumor growth in NF2 or during tumor resection. Disadvantages include a potentially more complicated surgical approach and the inability to resect an acoustic neuroma at the same sitting. The influence of stimulating the auditory system at a more highly processed level is unknown.

As with the cochlear nucleus, the tonotopy of the inferior colliculus is arranged perpendicular to its surface. A penetrating electrode array has been developed in association with Cochlear Corporation. This array consists of a single pin with 20 electrodes organized circumferentially along its length and is designed to be inserted via the supracerebellar, infratentorial route using an introducing stylet. ${ }^{26}$ The auditory midbrain implant is cur- 
rently undergoing initial evaluation in humans at Hannover, Germany.

\section{REFERENCES}

1. Edgerton BJ, House WF, Hitselberger W. Hearing by cochlear nucleus stimulation in humans. Ann Otol Rhinol Otolaryngol Suppl 1982;91:117-124.

2. Otto SR, Shannon RV, Brackmann DE, Hitselberger WE, Staller S, Menapace C. The multichannel auditory brain stem implant: performance in twenty patients. Otolaryngol Head Neck Surg 1998; 118:291-303.

3. Otto SR, Brackmann DE, Hitselberger WE, Shannon RV, Kuchta J. Multichannel auditory brainstem implant: update on performance in 61 patients. J Neurosurg 2002;96:1063-1071.

4. Otto S, Staller S. Multichannel auditory brain stem implant: case studies comparing fitting strategies and results. Ann Otol Rhinol Laryngol Suppl 1995;166:36-39.

5. Eisenberg LS, Maltan AA, Portillo F, Mobley JP, House WF. Electrical stimulation of the auditory brain stem structure in deafened adults. J Rehabil Res Dev 1987;24:9-22.

6. Brackmann DE, Hitselberger WE, Nelson RA, et al. Auditory brainstem implant: I. Issues in surgical implantation. Otolaryngol Head Neck Surg 1993;108:624-633.

7. Heller JW, Brackmann DE, Tucci DL, Nyenhuis JA, Chou CK. Evaluation of MRI compatibility of the modified nucleus multichannel auditory brainstem and cochlear implants. Am J Otol 1996;17:724-729.

8. Slattery WH 3rd, Brackmann DE, Hitselberger W. Hearing preservation in neurofibromatosis type 2. Am J Otol 1998;19: $638-642$.

9. Monsell EM, McElveen JT Jr, Hitselberger WE, House WF. Surgical approaches to the human cochlear nuclear complex. Am J Otol 1987;8:450-455.

10. Colletti V, Fiorino FG, Carner M, Giarbini N, Sacchetto L, Cumer G. The retrosigmoid approach for auditory brainstem implantation. Am J Otol 2000;21:826-836.

11. Waring MD. Intraoperative electrophysiologic monitoring to assist placement of auditory brainstem implant. Ann Otol Rhinol Laryngol Suppl 1995;166:33-36.

12. Shannon RV, Fayad J, Moore J, et al. Auditory brainstem implant: II. Postsurgical issues and performance. Otolaryngol Head Neck Surg 1993;108:634-642.
13. Shannon RV, Moore JK, McCreery DB, Portillo F. Thresholddistance measures from electrical stimulation of human brainstem. IEEE Trans Rehabil Eng 1997;5:70-74.

14. Finitzo-Hieber T, Gerling IJ, Matkin ND, Cherow-Skalka E. A sound effects recognition test for the pediatric audiological evaluation. Ear Hear 1980;1:271-276.

15. Erber NP, Alencewicz CM. Audiologic evaluation of deaf children. J Speech Hear Disord 1976;41:256-267.

16. Elliott LL, Katz DR. Development of a new children's test of speech discrimination. St. Louis, MO: Auditec of St. Louis, 1980 (audio format).

17. Giolas TG, Duff JR. Equivalency of CID and revised CID sentence lists. J Speech Hear Res 1973;16:549-555.

18. Tyler RS, Preece JP, Tye-Murray N. Iowa Audiovisual Speech Perception Laser Videodisc. Iowa City: University of Iowa, 1987 (video format).

19. Boothroyd A. CUNY Topic Related Sentence Test Laservideo Disk. New York: City University of New York, 1988 (video format).

20. Niparko JK, Altschuler RA, Xue XL, Wiler JA, Anderson DJ. Surgical implantation and biocompatibility of central nervous system auditory implants. Ann Otol Rhinol Laryngol 1989;98:965970.

21. El-Kashlan HK, Niparko JK, Altschuler RA, Miller JM. Direct electrical stimulation of the cochlear nucleus: surface versus penetrating stimulation. Otolaryngol Head Neck Surg 1991;105:533543.

22. McCreery DB, Shannon RV, Moore JK, Chatterjee M. Accessing the tonotopic organization of the ventral cochlear nucleus by intranuclear microstimulation. IEEE Trans Rehabil Eng 1998;6:391399.

23. McCreery DG, Yuen TGH, Bullara LA. Chronic microstimulation in the feline ventral cochlear nucleus: physiologic and histologic effects. Hearing Res 2000;149:223-238.

24. Colletti V, Shannon RV. Open set speech perception with auditory brainstem implant? Laryngoscope 2005;115:1974-1978.

25. Colletti V, Carner M, Miorelli V, Guida M, Colletti L, Fiorino F. Auditory brainstem implant (ABI); new frontiers in adults and children. Otolaryngol Head Neck Surg 2005;133:126-138.

26. Lenarz T, Lim HH, Reuter G, Patrick JF, Lenarz M. The auditory midbrain implant: a new auditory prosthesis for neural deafness - concept and device description. Otol Neurotol 2006; $27: 838-843$. 